\title{
Reply to the Letter: “Low levels of transaminase and mortality risk in older people with special reference to sarcopenia"
}

\author{
Paolo Gallo ${ }^{1}$ Antonio De Vincentis ${ }^{2}\left[\right.$ - Stefania Bandinelli ${ }^{3}$. Luigi Ferrucci ${ }^{4} \cdot$ Antonio Picardi $^{1}$. \\ Raffaele Antonelli Incalzi ${ }^{2}$. Umberto Vespasiani-Gentilucci ${ }^{1}$
}

Received: 4 October 2021 / Accepted: 6 November 2021 / Published online: 16 November 2021

(c) The Author(s), under exclusive licence to Springer Nature Switzerland AG 2021

\section{Dear Editor,}

We read with interest the comments by Dr. Tomoyuki Kawada on our recently published study [1], where, analyzing a cohort of 761 community-dwelling older subjects, we showed that the prognostic information conveyed by AST and ALT with respect to all-cause and cause-specific mortality is improved when the enzyme levels are considered together.

In this regard, Dr. Tomoyuki Kawada specifically argued on the possible confounding effect of sarcopenia and on the opportunity to introduce repeated measures of sarcopenia as independent variables in the context of a time-dependent analysis [2].

We agree with the point raised; indeed, we have presented an adjusted model including also sarcopenia, frailty, disability, and comorbidities [1]. Notably, the association of lower aminotransferases with poor outcomes was maintained even in this model, suggesting that also other unexplored confounders subtend this association [1]. Further well-designed studies aimed to explore these unrecognized determinants associated both with liver enzymes and with poor outcomes are warranted.

We agree also that a time-dependent approach with repeated measures of sarcopenia would be desirable, but

Antonio De Vincentis

a.devincentis@unicampus.it

1 Clinical Medicine and Hepatology Unit, Campus BioMedico University, Rome, Italy

2 Internal Medicine Unit, Campus Bio-Medico University, Via Alvaro del Portillo, 20000128 Rome, Italy

3 Geriatric Unit, Azienda Sanitaria di Firenze Toscana, Firenze, Italy

4 National Institute on Aging, National Institutes of Health, Baltimore, MD, USA that possibility has been hampered by the unavailability of these data.

Finally, literature [3] clearly supports sex-specific pathophysiological mechanisms for sarcopenia and sex has to be taken into account in studies exploring the association between low levels of transaminases, sarcopenia, and mortality. Actually, in our study, the associations observed were not significantly different between the two genders [1].

Compliance with Ethical Standard Not required.

Conflict of Interest Authors deny any conflict of interest.

Statement of human and animal rights Not required.

Informed consent Not required.

\section{References}

1. Gallo P, De Vincentis A, Bandinelli S et al (2021) Combined evaluation of aminotransferases improves risk stratification for overall and cause-specific mortality in older patients. Aging Clin Exp Res. https://doi.org/10.1007/s40520-021-01979-9

2. Kawada T (2021) Low levels of transaminase and mortality risk in older people with special reference to sarcopenia. Aging Clin Exp Res. https://doi.org/10.1007/s40520-021-02010-x

3. Tay L, Ding YY, Leung BP et al (2015) Sex-specific differences in risk factors for sarcopenia amongst community-dwelling older adults. Age (Dordr). https://doi.org/10.1007/s11357-015-9860-3

Publisher's Note Springer Nature remains neutral with regard to jurisdictional claims in published maps and institutional affiliations. 